\title{
Lípides e lipoproteínas séricos em crianças e adolescentes ambulatoriais de um hospital universitário público
}

\author{
Serum lipids and lipoproteins in children and adolescents from a public university hospital outpatient clinic
}

Eliana Cotta de Faria ${ }^{1}$, Fabio Bernardi Dalpino ${ }^{2}$, Raquel Takata ${ }^{3}$

\section{RESUMO}

Objetivo: Estabelecer a prevalência de dislipidemias em uma amostra populacional brasileira ambulatorial de crianças e adolescentes de um centro hospitalar terciário público.

Métodos: Estudo retrospectivo de análise de resultados de lípides e lipoproteínas de 1.937 indivíduos de ambos os sexos, com idades entre dois e 19 anos, atendidos no ambulatório do Hospital de Clínicas (HC) da Universidade Estadual de Campinas, no Estado de São Paulo, entre 2000 e 2007. Os valores de referência adotados foram os recomendados por Kwiterovich. A análise estatística utilizou o programa SPSS.

Resultados: Valores alterados de colesterol, LDL-colesterol e triglicérides foram encontrados em 44, 36 e $56 \%$ das crianças de dois a nove anos e em 44, 36 e 50\% dos adolescentes de dez a 19 anos, respectivamente. Foi observada redução de HDL-colesterol em $44 \%$ das crianças e $49 \%$ dos adolescentes. Hipercolesterolemia combinada com hipertrigliceridemia estava presente em $34 \%$ das crianças e dos adolescentes; hipercolesterolemia e hipoalfalipoproteinemia estavam presentes em $15 \%$ das crianças e $20 \%$ dos adolescentes; e hipertrigliceridemia e hipoalfalipoproteinemia ocorreram em $33 \%$ das crianças e $30 \%$ dos adolescentes.

Conclusões: Este estudo demonstrou alta prevalência de dislipidemias em crianças e adolescentes atendidos em um ambulatório de hospital universitário público, o que aponta para a necessidade de estudos em outras comunidades brasileiras, tanto hospitalares quanto não hospitalares, para o diagnóstico precoce e tratamento das dislipidemias e para a adoção de medidas preventivas com relação às doenças cardiovasculares associadas.

Palavras-chave: lipídeos; lipoproteínas; dislipidemias; crianças; adolescentes.

\section{ABSTRACT}

Objective: To establish the prevalence of dyslipidemia among children and adolescents assisted at public university hospital outpatient clinic

Methods: This retrospective study analyzed the lipid profile of 1,937 outpatients of both genders, from two to 19 years old, assisted at the outpatient clinic of the Clinical Hospital of the State University of Campinas, Brazil, from 2000 to 2007. The cutoff values adopted were those recommended by Kwiterovich. Statistical analysis used the SPSS software.

Results: Altered values of cholesterol, LDL-cholesterol and triglycerides were found in 44,36 and $56 \%$ of the children (2-9 years old) and in 44, 36 and $50 \%$ of the adolescents (10-19 years old), respectively. Reduced HDL-cholesterol concentrations were present in $44 \%$ of the children and $49 \%$ of the adolescents. A combination of hypercholesterolemia and hypertriglyceridemia was present in $34 \%$ of children and adolescents. Hypercholesterolemia combined with hypoalphalipoproteinemia was present in $15 \%$ of the children and $20 \%$ of the adolescents, and hypertriglyceridemia combined with hypoalphalipoproteinemia was present in $33 \%$ of the children and $30 \%$ of the adolescents.
Professora-associada do Departamento de Patologia Clínica da Faculdade de Ciências Médicas da Universidade Estadual de Campinas (FCM-Unicamp), Campinas, SP, Brasil

2Doutor em Ciências Médicas, na área de Patologia Clínica, pela FCMUnicamp, Campinas, SP, Brasil

${ }^{3}$ Mestre em Ciências Médicas, na área de Patologia Clínica, pela FCMUnicamp, Campinas, SP, Brasil

Endereço para correspondência:

Eliana Cotta de Faria

\section{Departamento de Patologia Clínica da FCM-Unicamp \\ Caixa Postal 6111 \\ CEP 13083-970 - Campinas/SP \\ E-mail: cotta@fcm.unicamp.br}

Fonte financiadora do projeto: Fundação de Amparo à Pesquisa do Estado de São Paulo (Fapesp) e Conselho Nacional de Desenvolvimento Científico e Tecnológico (CNPq)

Recebido em: 10/10/2007

Aprovado em: 20/1/2008 
Conclusions: The frequency of dyslipidemias in children and adolescents of the out-patient clinic of a public university hospital was high. There is a need to further study hospital and non-hospital communities in order to establish early diagnosis and treatment of dyslipidemias and to avoid associated cardiovascular diseases.

Key-words: lipids; lipoproteins; dyslipidemia; children; adolescents.

\section{Introdução}

As manifestações clínicas da doença arterial coronariana surgem, em geral, na idade adulta. Entretanto, diversos estudos têm demonstrado que a doença aterosclerótica da camada íntima-média arterial surge na infância, de forma silenciosa, progredindo significativamente a partir da terceira década de vida ${ }^{(1-3)}$.

As dislipidemias são os fatores de risco mais importantes para o desenvolvimento da aterosclerose e de suas complicações e têm sido objeto de estudos em crianças e adolescentes. Isto se dá tanto pela alta prevalência encontrada nesta faixa etária (dois a 19 anos), quanto pela identificação de que a colesterolemia na infância é um fator preditor da lipemia na idade adulta, sendo a doença arterial coronariana a principal causa de morbimortalidade no Brasil ${ }^{(2,4,5)}$.

As análises de lípides e lipoproteínas séricos são indispensáveis para o diagnóstico das dislipidemias e para a prevenção primária de doença arterial coronariana ${ }^{(6)}$. Consensos internacionais, como o National Cholesterol Education Program (NCEP) ${ }^{(7)}$, visam estabelecer medidas profiláticas e formas de tratamento adequadas para a doença. A IV Diretriz Brasileira sobre Dislipidemias (IV DBSD $)^{(8)}$ adota os valores recomendados pelo NCEP, mas ainda há discussão sobre os pontos de corte e se outros valores de referência, como o de Kwiterovich ${ }^{(9)}$, podem ser adotados

São poucos os estudos realizados no nosso país para estabelecer o perfil lipídico de crianças e adolescentes, mas os que existem demonstraram grande proporção de indivíduos com níveis de colesterol acima do recomendado ${ }^{(10,11)}$. Assim, o objetivo deste estudo foi estabelecer a prevalência de dislipidemias em uma amostra populacional ambulatorial de crianças e adolescentes de um centro terciário hospitalar entre 200 e 2007.

\section{Métodos}

Trata-se de um estudo retrospectivo relativo ao período de 2000 a 2007, envolvendo 1.937 crianças e adolescentes de dois a 19 anos de idade, de ambos os sexos, de diferentes classes socioeconômicas, atendidas em todos os ambulatórios do Hospital de Clínicas da Universidade Estadual de Campinas (HC-Unicamp), Campinas, São Paulo.

Foram avaliados os resultados dos seguintes exames laboratoriais: colesterol total (CT), HDL-colesterol (HDL-C), LDL-colesterol (LDL-C) e triglicérides (TG). Após coleta matutina em jejum de 10-12 horas, os exames foram realizados na Seção de Bioquímica Clínica da Divisão de Patologia Clínica, em analisadores químicos automáticos (Hitachi 917, Roche), utilizando-se métodos e reagentes da Roche Diagnostics. Foram empregados sistematicamente materiais controle (Precinorm ${ }^{\circledR}$ e Precipath ${ }^{\circledR}$, Roche Diagnostics) para a determinação da variabilidade analítica e manutenção da acurácia.

Os valores de corte utilizados foram os recomendados por Kwiterovich ${ }^{(9)}$ : CT $<170 \mathrm{mg} / \mathrm{dL}, \mathrm{HDL}-\mathrm{C}>45 \mathrm{mg} / \mathrm{dL}$, LDL-C $<110 \mathrm{mg} / \mathrm{dL}$ e TG $<75 \mathrm{mg} / \mathrm{dL}$ até dez anos e $<90 \mathrm{mg} /$ $\mathrm{dL}$ entre dez e 19 anos.

O conjunto de resultados de exames deste estudo foi gerado no programa de base de dados do Sistema Informatizado do HC-Unicamp, desenvolvido pelo Núcleo de Informática do HC. Considerou-se apenas o primeiro resultado do exame por ano para cada participante. Informações sobre a identificação hospitalar, sexo, idadee data da coleta também constam deste banco de dados. Pelo seu caráter retrospectivo, não foi possível controlar variáveis como dieta, atividade física ou influência de medicamentos.

O cálculo da prevalência de dislipidemias foi baseado no número de participantes em cada grupo: amostra total de 1.937 indivíduos, sendo 858 do sexo masculino, 1.079 do sexo feminino, 578 entre dois a nove anos e 1.359 de dez a 19 anos. Os dados obtidos foram constituídos por 2.962 dosagens de CT, 2.629 de HDL-C, 2.504 de LDL-C e 2.886 de TG, num total de 10.981 dosagens.

As análises descritivas paramétricas e não paramétricas e testes comparativos foram realizados utilizando-se o programa Statistical Package for the Social Sciences (SPSS, Chicago, EUA, versão 6.0). O projeto de pesquisa foi aprovado pelo Comitê de Ética em Pesquisa da Faculdade de Ciências Médicas da Unicamp.

\section{Resultados}

Foram avaliados 1.937 indivíduos, com idade média de $13 \pm 5$ anos, sendo $44 \%$ do sexo masculino e $56 \%$ do feminino. Os coeficientes de variação interensaios para cada parâmetro estavam abaixo do erro total recomendado pelo $\operatorname{NCEP}^{(12,13)}$. 
$\mathrm{Na}$ população estudada, as concentrações médias e as medianas variações dos parâmetros analisados foram, respectivamente: $178 \pm 73 \mathrm{mg} / \mathrm{dL}$ e 165 (39-1033) $\mathrm{mg} / \mathrm{dL}$ para CT; $48 \pm 15 \mathrm{mg} / \mathrm{dL}$ e $46(4-129) \mathrm{mg} / \mathrm{dL}$ para HDLC; $108 \pm 55 \mathrm{mg} / \mathrm{dL}$ e $99(6-792) \mathrm{mg} / \mathrm{dL}$ para LDL-C e $117 \pm 135 \mathrm{mg} / \mathrm{dL}$ e $84(18-2640) \mathrm{mg} / \mathrm{dL}$ para TG. A análise por sexo mostrou valores médios mais elevados no sexo feminino para HDL-C $(p<0,05)$ e, no sexo masculino, para TG $(p<0,05)$. Os níveis de CT e LDL-C não variaram por sexo. Com relação à idade, o HDL-C não apresentou variação; os demais parâmetros analisados apresentaram redução significativa na faixa etária de dez a 19 anos, comparados aos encontrados nas crianças de dois a nove anos de idade (Tabela 1).

$\mathrm{Na}$ Tabela 2, estão demonstradas as freqüências de dislipidemias ao longo do estudo, de acordo com o sexo e a faixa etária. A hipertrigliceridemia foi a dislipidemia mais freqüente. A hipoalfalipoproteinemia foi predominante no sexo masculino, ocorrendo em $54 \%$ dos meninos.
A dislipidemia mista mais freqüente na população estudada foi o aumento de CT combinado com TG, encontrada em $34 \%$ dos indivíduos. No sexo masculino, predominou a dislipidemia mista com TG alto e HDL-C baixo e, no sexo feminino e em ambas as faixas etárias, houve predomínio de CT e TG altos (Tabela 3).

\section{Discussão}

No Brasil, de acordo com dados do Sistema Único de Saúde (SUS) $^{(14)}$, as doenças do aparelho circulatório representam $27 \%$ de todas as causas de morte para todas as idades. Dessa forma, é importante estabelecer a prevalência de dislipidemias nas faixas etárias estudadas no presente estudo, pois o processo aterosclerótico inicia-se na infância ${ }^{(1-3)}$. Observa-se grande carência desses estudos no Brasil, apesar de sua ampla importância no país.

Como as manifestações clínicas da doença não ocorrem precocemente em crianças e adolescentes, é difícil estabelecer uma relação entre doença arterial coronariana

Tabela 1 - Médias, medianas e valores mínimos e máximos das concentrações séricas (mg/dL) de lípides e lipoproteínas, segundo sexo e faixas etária

\begin{tabular}{lccccc}
\hline Parâmetro & Total & Masculino & Feminino & 2 a 9 anos & 10 a 19 anos \\
\hline CT & $178 \pm 73$ & $176 \pm 81$ & $180 \pm 66$ & $183 \pm 89$ & $174 \pm 67^{*}$ \\
HDL-C & $165(39-1033)$ & $161(39-990)$ & $168(46-1033)$ & $165(39-927)$ & $165(74-1033)$ \\
& $48 \pm 15$ & $47 \pm 15$ & $49 \pm 14^{\dagger}$ & $48 \pm 15$ & $48 \pm 15$ \\
LDL-C & $46(4-129)$ & $45(9-129)$ & $48(4-126)$ & $46(11-116)$ & $46(4-129)$ \\
& $108 \pm 55$ & $107 \pm 64$ & $109 \pm 47$ & $112 \pm 64$ & $106 \pm 51^{*}$ \\
TG & $99(6-792)$ & $96(6-792)$ & $101(8-556)$ & $102(6-717)$ & $98(8-792)$ \\
& $117 \pm 135$ & $122 \pm 159^{\ddagger}$ & $112 \pm 110$ & $123 \pm 174$ & $114 \pm 117^{*}$ \\
& $84(18-2640)$ & $84(18-2640)$ & $85(18-2520)$ & $82(18-2640)$ & $85(18-2520)$ \\
\hline
\end{tabular}

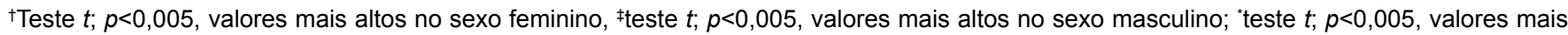
baixos na faixa etária de 10 a 19 anos

Tabela 2 - Freqüências percentuais de dislipidemias (valores aumentados de CT ou LDL-C ou TG ou redução de HDL-C), segundo sexo e faixa etária

\begin{tabular}{lccccc}
\hline Tipo/grupo & Total & Masculino & Feminino & 2 a 9 anos & 10 a 19 anos \\
\hline CT & 44 & 40 & 48 & 44 & 44 \\
HDL-C & 48 & 54 & 43 & 44 & 49 \\
LDL-C & 36 & 33 & 39 & 36 & 36 \\
TG & 52 & 50 & 53 & 56 & 50 \\
\hline
\end{tabular}

Tabela 3 - Freqüências percentuais de dislipidemias mistas (combinação de valores aumentados para CT ou TG com redução de HDL-C ou valores aumentados para CT e TG), segundo sexo e faixas etária

\begin{tabular}{lccccc}
\hline Tipo/grupo & Total & Masculino & Feminino & 2 a 9 anos & 10 a 19 anos \\
\hline CT+TG & 34 & 33 & 35 & 34 & 34 \\
CT+HDL-C & 18 & 18 & 19 & 15 & 20 \\
TG+HDL-C & 31 & 34 & 29 & 33 & 30 \\
\hline
\end{tabular}


Tabela 4 - Freqüências percentuais de dislipidemias de crianças e adolescentes: estudos da literatura

\begin{tabular}{lcccc}
\hline Autor (país/ano) & CT $^{\mathbf{a}}$ & HDL-C $^{\mathbf{b}}$ & LDL-C $^{\mathbf{c}}$ & TG $^{\mathbf{d}}$ \\
\hline Presente estudo (Brasil/2007) & 44 & 48 & 36 & 52 \\
Scherr et a/(17) (Brasil/2007) & 49 & nd & nd & nd \\
Romaldini et a/(2) (Brasil/2004) & 28 & 14 & 19 & 13 \\
Reinehr et a/(21) (Europa/2004) & 11 & 11 & 9 & 29 \\
Gerber e Zielinsky (20) (Brasil/2003) & 28 & nd & nd & nd \\
Moura et al/(4) (Brasil/2000) & 35 & nd & nd & nd \\
\hline
\end{tabular}

${ }^{a} \mathrm{C} T=$ hipercolesterolemia; ${ }^{\mathrm{H}} \mathrm{HDL}-\mathrm{C}=$ hipoalfalipoproteinemia; ${ }^{\mathrm{L}} \mathrm{LDL}-\mathrm{C}=$ hiperbetalipoproteinemia; ${ }^{\mathrm{d}} \mathrm{TG}=$ hipertrigliceridemia; nd=não determinado

e concentrações séricas de lípides nessas faixas etárias. Entretanto, estudos baseados em autópsias revelam o surgimento de estrias gordurosas na camada íntima das artérias coronárias a partir dos dez anos de idade ${ }^{(1,15)}$. Cumpre citar que a prevalência da síndrome metabólica em crianças e adolescentes cresceu muito na última década, com aumento nas taxas de sobrepeso e obesidade desde crianças entre dois e cinco anos ${ }^{(16)}$.

O presente estudo foi realizado em ambulatório de um hospital universitário do Estado de São Paulo, que atende a cerca de 115 mil pacientes por ano, provenientes de mais de cem municípios, correspondendo a uma população de cerca de 5.000 .000 de pessoas. Com base nos critérios recomendados por Kwiterovich ${ }^{(11)}$ para crianças e adolescentes, observou-se alta prevalência de dislipidemias: $44 \%$ dos indivíduos com hipercolesterolemia, $48 \%$ com hipoalfalipoproteinemia, $36 \%$ com hiperbetalipoproteinemia e $52 \%$ com hipertrigliceridemia. Também foi notada alta freqüência de dislipidemias mistas nas crianças e adolescentes avaliados. Além disso, as concentrações médias de CT e TG encontravam-se acima dos valores recomendados, assim como o LDL-C na faixa etária de dois a nove anos.

Alguns trabalhos realizados com pacientes brasileiros também encontraram alta freqüência de dislipidemias nessas faixas etárias; porém, neste estudo, os valores foram 1,3 a 5 vezes maiores (Tabela 4). As dislipidemias no grupo infanto-juvenil são mais freqüentes provavelmente devido às mudanças nos hábitos alimentares associadas à redução na prática de atividades físicas, com estabelecimento de vida sedentária.

Romaldini et $a l^{(2)}$, avaliando os fatores de risco para aterosclerose em 109 crianças e adolescentes com história familiar de doença arterial coronariana prematura, identificaram que $41 \%$ dos participantes apresentavam um a quatro fatores de risco: $28 \%$ tinham CT $>170 \mathrm{mg} / \mathrm{dL}$, $19 \% \mathrm{LDL}-\mathrm{C}<110 \mathrm{mg} / \mathrm{dL}, 14 \% \mathrm{HDL}-\mathrm{C}<40 \mathrm{mg} / \mathrm{dL}$ e $13 \% \mathrm{TG}>130 \mathrm{mg} / \mathrm{dL}$.
Moura $e t a l^{(4)}$, ao estudarem 1.600 escolares de sete a 14 anos da cidade de Campinas, encontraram freqüência elevada de hipercolesterolemia: $35 \%$ da amostra populacional estudada apresentava CT $>170 \mathrm{mg} / \mathrm{dL}$.

Scherr $e t a l^{(17)}$ analisaram crianças que freqüentavam escolas públicas e privadas na cidade do Rio de Janeiro e observaram valores de CT, HDL-C e LDL-C mais elevados nos estudantes de escolas privadas, dos quais $49 \%$ apresentavam $\mathrm{CT}>170 \mathrm{mg} / \mathrm{dL}$. Houve diferença da dieta e da atividade física praticadas por estas crianças, em comparação àquelas que freqüentavam escolas públicas.

Elias et $a l^{(18)}$ avaliaram 43 adolescentes filhos de hipertensos e encontraram as seguintes médias para: $\mathrm{CT}, 177 \mathrm{mg} / \mathrm{dL}$, LDL-C, $119 \mathrm{mg} / \mathrm{dL}$, HDL-C, 39mg/dL e TG, 99mg/dL. Nesse estudo, maiores níveis de pressão arterial e médias mais elevadas no perfil lipídico ocorreram em filhos de hipertensos, comparados aos filhos de normotensos, comprovando a necessidade de avaliar lípides e lipoproteínas em crianças e adolescentes assintomáticos, especialmente quando existe na família antecedente de fatores de risco para a doença arterial coronariana.

Estudo americano ${ }^{(19)}$ demonstrou que $25 \%$ das crianças apresentavam colesterolemia acima de $170 \mathrm{mg} / \mathrm{dL}$ e, no Brasil, alguns trabalhos também têm mostrado alta prevalência dessa alteração, destacando-se o de Gerber e Zielinsky ${ }^{(20)}$, no qual se nota freqüência de $28 \%$ de colesterol alto em crianças entre seis e 14 anos.

No estudo de Reinehr $e t a^{(21)}$ foi descrita alta prevalência de fatores de risco para doença cardiovascular em crianças e adolescentes obesos, observando-se 11,9 e $29 \%$, respectivamente, de indivíduos com CT, LDL-C e TG elevados e $11 \%$ com redução no HDL-C.

Existe uma associação de fatores ambientais e genéticos na etiologia das dislipidemias, sendo os genéticos de grande importância. Gulati e Saxena ${ }^{(1)}$ avaliaram crianças indianas de cinco a 14 anos cujos parentes tinham antecedentes de 
doença arterial coronariana prematura. Ao confrontar os achados com um grupo controle, sem história familiar de doença arterial coronariana, os autores encontraram diferenças significativas entre os dois grupos. Observou-se níveis mais elevados de CT, LDL-C e TG e valores reduzidos de HDL-C no grupo com história pregressa de doença arterial coronariana comparado ao grupo controle. Além disso, houve correlação positiva entre os valores de lípides do parente com doença arterial coronariana, prematura e a sua criança.

\section{Referências bibliográficas}

1. Gulati S, Saxena A. Study of lipid profile in children of patients with premature coronary artery disease. Indian Pediatr 2003;40:556-60.

2. Romaldini CC, Issler H, Cardoso AL, Diament J, Forti N. Risk factors for atherosclerosis in children and adolescents with family history of premature coronary artery disease. J Pediatr (Rio J) 2004;80:135-40.

3. Coronelli CLS, Moura EC. Hypercholesterolemia and risk factors among school children. Rev Saude Publica 2003;37:24-31.

4. Moura EC, Castro CM, Mellin AS, Figueiredo DB. Lipidic profile among school children, Brazil. Rev Saude Publica 2000;34:499-505.

5. Santos RD, Spósito AC, Santos JE, Fonseca FH, Moriguchi EH, Martinez TLR et al. PANDORA - Survey of Brazilian cardiologists about cholesterol reduction. Arq Bras Cardiol 2000;75:289-302.

6. Seki M, Seki MO, Niyama FP, Pereira Junior PG, Seki MO, Matsuo T et al. Determination of lipid and lipoprotein classification levels in school children from ten to nineteen years old from the city of Maracaí, SP, Brazil. J Bras Patol Med Lab 2003;39:309-16.

7. Autoria não referida. Third report of the National Cholesterol Education Program (NCEP) expert panel on detection, evaluation, and treatment of high blood cholesterol in adults (adult treatment panel iii) final report. Circulation 2002;106:3143-421.

8. Sposito AC, Caramelli B, Fonseca FA, Bertolami MC, Afiune Neto A,Souza AD et al. IV Brazilian guideline for dyslipidemia and atherosclerosis prevention: Department of Atherosclerosis of Brazilian Society of Cardiology. Arq Bras Cardiol 2007;88 (Suppl 1):2-19.

9. Kwiterovich PO. Plasma lipid and lipoprotein levels in childhood. Ann NY Acad Sci 1991;623:90-107.

10. Martinez TLR, Santos RD, Armaganijan D, Torres KP, Loures-Vale A, Magalhães $\mathrm{ME}$ et al. National alert campaign about increased cholesterol. Determination of cholesterol levels in 81,262 Brazilians. Arq Bras Cardiol 2003;80:635-8.

11. Rabelo LMR, Viana RM, Schimith MA, Patin RV, Valverde MA, Denadai RC et al. Risk factors for atherosclerosis in students of a private university in São Paulo-Brazil. Arq Bras Cardiol 1999;72:569-80.
O presente estudo aponta para a necessidade do desenvolvimento de outras pesquisas na faixa etária inferior a 20 anos e em outros grupos populacionais para o diagnóstico e o tratamento das dislipidemias. Além disto, há necessidade da adoção de medidas preventivas com relação às doenças cardiovasculares, como programas educacionais nas escolas para as crianças e suas famílias, os quais devem enfatizar a importância de hábitos alimentares saudáveis e da prática de atividades físicas regulares.
12. Autoria não referida. Medicare, Medicaid and CLIA programs; revision of the laboratory regulations for the Medicare, Medicaid, and Clinical Laboratories Improvement Act of 1967 programs--HCFA. Final rule with comment period. Fed Regist 1990;55:9538-610.

13. Autoria não referida. Medicare program; Medicare and laboratory certification program. Enforcement procedures for laboratories-HCFA. Final rule. Fed Regist 1992;57:7218-43.

14. Brasil - Ministério da Saúde - DATASUS [homepage on the Internet]. Informações de saúde. Morbidade e informações epidemiológicas [cited 2005 May 18]. Available from: http://tabnet.datasus.gov.br/cgi/tabcgi. exe?sim/cnv/obtuf.def

15. Strong JP, McGill HC Jr. The pediatric aspect of atherosclerosis. J Atheroscler Res 1969;9:251-65.

16. Chen W, Berenson GS. Metabolic syndrome: definition and prevalence in children. J Pediatr (Rio J) 2007;83:1-2.

17. Scherr C, Magalhães CK, Malheiros W. Lipid profile analysis in school children Arq Bras Cardiol 2007;89:65-70.

18. Elias MC, Bolívar MSM, Fonseca FAH, Martinez TLR, Angelini A, Ferreira C et al. Comparison of the lipid profile, blood pressure, and dietary habits of adolescents and children descended from hypertensive and normotensive individuals. Arq Bras Cardiol 2004;82:139-42.

19. No authors listed. Cholesterol screening in children. U.S. Public Health Service. Amer Fam Physician 1995;51:1923-7.

20. Gerber ZRS, Zielinsky P. Fatores de risco de aterosclerose na infância: um estudo epidemiológico. Arq Bras Cardiol 1997;69:231-6.

21. Reinehr T, Wabitsch M, Andler W, Beyer P, Bottner A, Chen-Stute A et al. Medical care of obese children and adolescents. APV: a standardised multicentre documentation derived to study initial presentation and cardiovascular risk factors in patients transferred to specialised treatment institutions. Eur J Pediatr 2004;163:308-12. 